\title{
EFFECT OF FLUORIDE PROPHYLACTIC AGENTS ON CERAMIC SELF LIGATING BRACKETS
}

\author{
Yasser L Abdelnaby, ${ }^{1}$ Mahasen Taha $^{2}$ \\ ABSTRACT: \\ Purpose: To evaluate the effect of fluoride prophylactic \\ agents on surface roughness (Ra) and fracture load (FL) of \\ polycrystalline alumina bracket self ligating assembly. \\ Materials and Methods: Fifty maxillary central incisors self- \\ ligation polycrystalline alumina brackets (Damon Clear) were used \\ in this study. Brackets were divided into five equal groups. In \\ group 1; specimens were immersed in distilled water (dH2O) for 8 \\ hours. In group 2; specimens were immersed in 1.1\% sodium \\ fluoride solution ( $\mathcal{N a F}$ ) for 4 hours then in $d \mathcal{H} 2 \mathrm{O}$ for another \\ 4 hours. In group 3; specimens were immersed in $\mathcal{N a F}$ solution for \\ 8 hours. In groups 4 and 5 the same procedures were followed as \\ in group 2 and 3 respectively but 1.1\% acidulated phosphate \\ fluoride solution (APF) was used instead of $\mathcal{N a F}$. Finally, $R a$ \\ and FL of the self ligating assembly of the brackets were assessed. \\ The data was subjected to analysis of variance (ANOVA) and \\ Tukey post hoc tests. \\ Results: Vtilization of APF for 8 hours provided a \\ significantly higher Ra value than the other studied protocols \\ $(\mathcal{P}<.05)$. The later showed no significant differences between \\ them $(P>.05)$. No significant difference was found in $F \mathcal{L}$ among \\ different studied groups ( $P>.05$ ) except between brackets stored in \\ dH 20 and those stored in APF for 8 hours $(\mathcal{P}<.05)$.
}

1- Associate Professor, Orthodontic Department, Faculty of Dentistry, Mansoura University.

2- Lecturer, Orthodontic Department, Faculty of Dentistry, Mansoura University. 
Egyptian

Orthodontic Journal

Conclusion: APF should not be used for long periods with polycrystalline ceramic self ligating bracket because it adversely affects $R a$ and $F \mathcal{L}$ of the ligature assembly. On the other hand, $\mathcal{N a F}$ could be used safely.

\section{INTRODUCTION}

An important aspect in orthodontic practice is the preservation of tooth structures status during treatment. White spot lesions (WSLs) or demineralization areas have been reported in as many as $50 \%$ of orthodontic patients. ${ }^{1-5}$ They can appear in as few as 2-3 weeks after plaque accumulation around the brackets, particularly in patients with poor oral hygiene. ${ }^{6,7}$ Fluoride regimens such as professional fluoride application, self applied gel, fluoride containing tooth paste and fluoride mouth rinses have been recommended for patients undergoing orthodontic treatment. However, topical fluoride agents may act as corrosive agents, producing surface damage and strength reduction in dental materials such as orthodontic alloys, composite resins, and dental ceramics. $^{8-13}$

Since self ligating brackets (SLBs) have been pioneered in $1935,{ }^{14}$ they have undergone a revival with a variety of metal and ceramic designs. However, ceramic SLBs were introduced with metal ligating mechanics. ${ }^{15-17}$ Recently, a totally ceramic SLB (Damon Clear) was introduced to improve esthetics. The bracket body and slide (ligating mechanic) are made of study polycrystalline alumina.

Surface roughness and fracture strength of the brackets are of great importance as they could affect the efficiency of orthodontic treatment. Increased surface roughness exaggerates the frictional force between brackets and archwires which adversely affect the orthodontic treatment. ${ }^{18-21}$ In addition it could negatively affect the fracture resistance of the brackets. ${ }^{22-24}$ Fracture of ligature assembly is of great concern for orthodontist as the bracket becomes ineffective and ligation of the archwire to the bracket is no longer available. Several studies have examined fracture strength and roughness of ceramic brackets. ${ }^{22-26}$ Bracket tie wing was the main target of most of these studies. ${ }^{25,26}$ However, there are differences in shape and thickness between tie wing of traditional bracket and ligating assembly of self ligating bracket. 
Egyptian

Orthodontic Journal

Therefore the purpose of this study was to evaluate the effect of sodium fluoride $(\mathrm{NaF})$ and acidulated phosphate fluoride (APF) solutions on the surface roughness $(\mathrm{Ra})$ and fracture load $(\mathrm{FL})$ of polycrystalline alumina self ligating bracket assembly.

\section{MATERIAL AND METHODS}

A total of fifty maxillary right central incisors self-ligation polycrystalline alumina brackets with 0.022-inch slots (Damon Clear, ORMCO, Orange, Calif, USA) were used in this study. Brackets were divided into five equal groups, 10 specimens each. In group 1; specimens were immersed in distilled water $\left(\mathrm{dH}_{2} \mathrm{O}\right)$ for 8 hours. In group 2; specimens were immersed in $1.1 \% \mathrm{NaF}$ solution $(0.5 \% \mathrm{w} / \mathrm{v}$ fluoride, $\mathrm{pH}$ 7) for 4 hours then in distilled water for another 4 hours. In group 3; specimens were stored in $\mathrm{NaF}$ solution for 8 hours. In groups 4 and 5 the same procedure were followed as in group 2 and 3 respectively but $1.1 \%$ APF $(0.5 \% \mathrm{w} / \mathrm{v}$ fluoride, $\mathrm{pH} 5.1)$ was used instead of sodium fluoride solution. Specimens were stored at $37 \pm 1^{\circ} \mathrm{C}$. Finally, all brackets were assessed for Ra then FL.

\section{Roughness Testing:}

Surface roughness was evaluated using atomic force microscope (AUTOPROBE CP-Research, model AP-0100, THERMOMICROSCOBES, Calif, USA). The atomic force microscope (AFM) was used with the following specifications; scan rate: $1 \mathrm{~Hz}$, resolution: 256 x 256 line, used probe: contact ultralevers, used software: proscan 1.8 and software for image processing: IP2.1. Two areas, $25 \times 25 \mu^{2}$ each, of the labial surface of the ligature sliding cap were assessed. Average roughness (Ra) was evaluated in the present study.

\section{Fracture Load Testing:}

Brackets were bonded to flat acrylic surfaces which were fabricated from autopolymerizing acrylic resin (Duracryl, SpofaDental-Praha, Czech Republic) poured in plastic rings. The acrylic surfaces were roughened using diamond bur. Ceramic primer (ORMCO, USA) was applied to the acrylic and bracket base. Transbond XT primer (3M Unitek, Monrovia, Calif) was coated on the acrylic surface and bracket base. Transbond XT adhesive paste (3M Unitek) was applied to the base of the bracket and pressed onto the center of the acrylic surface. To avoid 
bracket debonding before bracket fracture, adhesive paste was added over the bracket base and flowed onto the acrylic surface. However, no adhesive was flowed under the sliding cap, bracket ears or into the arch wire slot. Then, the adhesive was light-cured on each interproximal side for 10 seconds.

Testing of FL was carried out using a computer controlled materials testing machine (Model LRX-plus; Lloyd Instruments Ltd., Fareham, UK). Each sample was mounted on the lower fixed compartment of the machine. A 0.014-inch stainless steel ligature wire (ORMCO, USA) was looped under closed ligature sliding cap and attached to the upper movable compartment of testing machine (Figure 1). Tensile load was applied at $10 \mathrm{~mm} / \mathrm{min}$ cross-head speed until bracket fracture occurred (Figure 2). The load required to fracture each bracket was recorded in Newton $(\mathrm{N})$. The data were recorded using computer software (Nexygen-MT; Lloyd Instruments).

Mean and standard deviations of Ra and FL measurements were calculated for all groups. The obtained data were subjected to analysis of variance (ANOVA) and Tukey post hoc tests to determine the significant difference among groups. Significance for all statistical tests was predetermined at $P<0.05$.

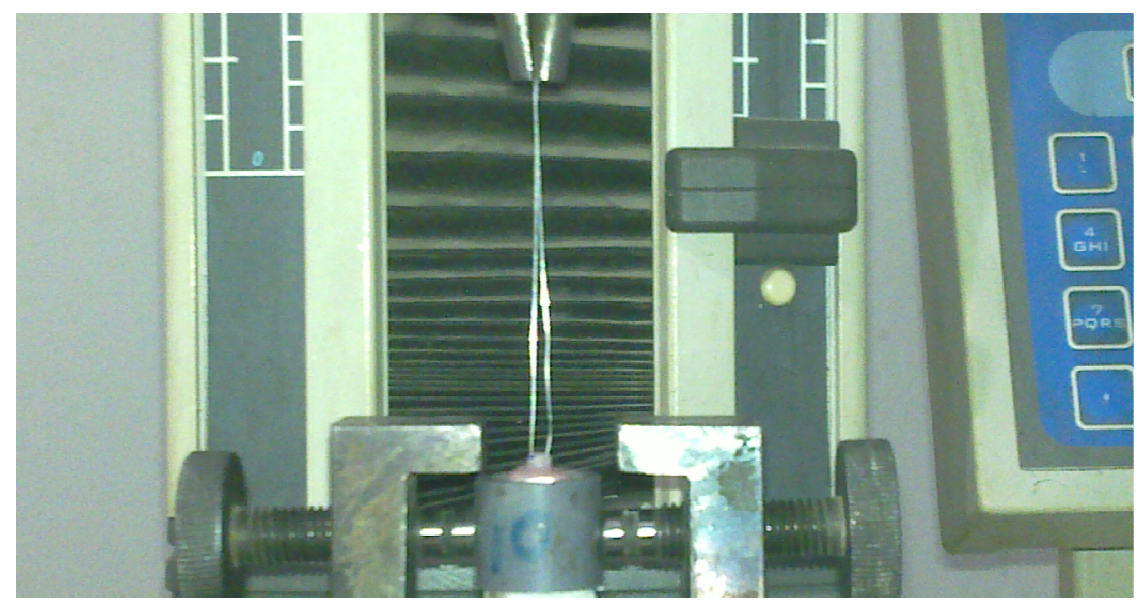

Figure 1: Specimen fixed to testing machine for assessment of fracture load of the self ligature assembly. 


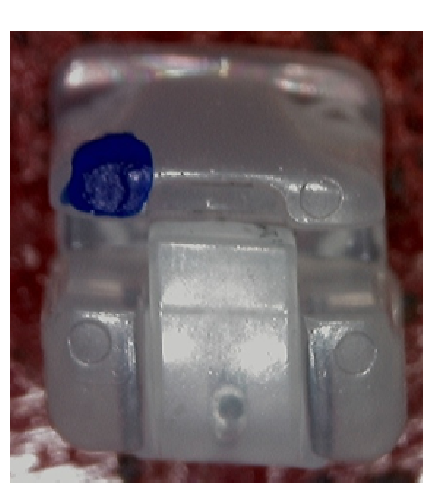

a

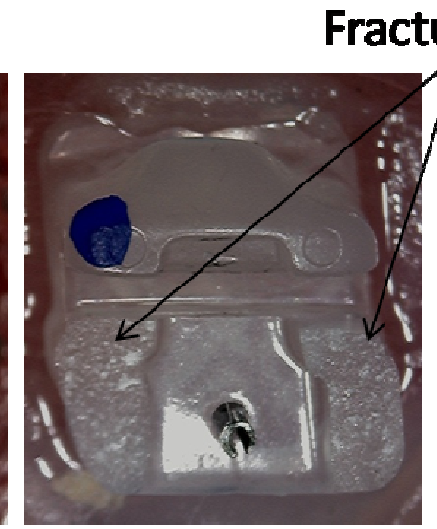

b

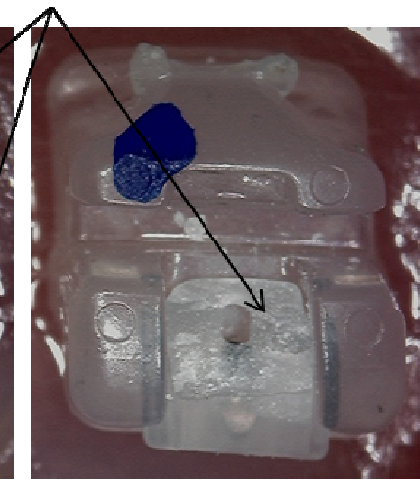

C

Figure 2: Polycrystalline ceramic self ligating bracket; a. intact, b. fractured at the bracket ears, c. fractured at sliding ligature cap.

Table I: Means and standard deviations of surface roughness and fracture load of the studied groups.

\begin{tabular}{lcc}
\hline \multicolumn{1}{c}{$\begin{array}{c}\text { Brackets immersed in different } \\
\text { solution }\end{array}$} & $\begin{array}{c}\text { Surface Rougness } \\
(\boldsymbol{\mu m})\end{array}$ & $\begin{array}{c}\text { Fracture Load } \\
(\mathbf{N})\end{array}$ \\
\hline $\mathrm{dH}_{2} \mathrm{O}$ for 8 hours & $0.053 \pm 0.006^{\mathrm{A}}$ & $74.58 \pm 3.93^{\mathrm{A}}$ \\
$\mathrm{NaF}$ for 4 hours $+\mathrm{dH}_{2} \mathrm{O}$ for 4 hours & $0.058 \pm 0.005^{\mathrm{A}}$ & $68.6 \pm 5.63^{\mathrm{AB}}$ \\
$\mathrm{NaF}$ for 8 hours & $0.059 \pm 0.006^{\mathrm{A}}$ & $67.2 \pm 4.36^{\mathrm{AB}}$ \\
$\mathrm{APF}$ for 4 hours $+\mathrm{dH}_{2} \mathrm{O}$ for 4 hours & $0.063 \pm 0.01^{\mathrm{A}}$ & $63.82 \pm 5.19^{\mathrm{AB}}$ \\
$\mathrm{APF}$ for 8 hours & $0.091 \pm 0.017^{\mathrm{B}}$ & $54.82 \pm 3.91^{\mathrm{B}}$ \\
\hline
\end{tabular}

Means with the same superscripted letters in same column are not significantly different at $\mathrm{P}<0.05$ according Tukey test. 


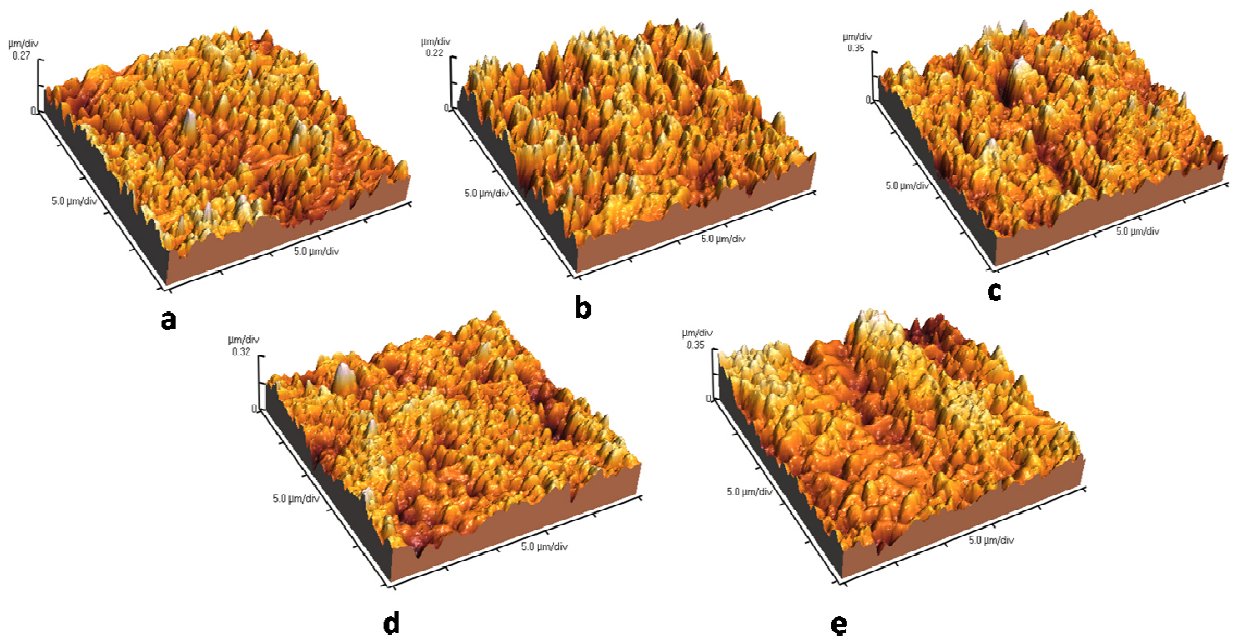

Figure 3: Three dimensional AFM images of the of ligature sliding cap of the studied groups; a. $\mathrm{dH}_{2} \mathrm{O}$ for 8 hours, b. $\mathrm{NaF}$ for 4 hours $+\mathrm{dH}_{2} \mathrm{O}$ for 4 hours, c. $\mathrm{NaF}$ for 8 hours, d.APF for 4 hours $+\mathrm{dH}_{2} \mathrm{O}$ for 4 hours, e. APF for 8 hours.

\section{RESULTS}

Means and standard deviations of the $\mathrm{Ra}$ and FL and results of Tukey post hoc test are expressed in Table I. AFM images (3D) of the labial surface of the bracket ligature sliding cap are presented in Figure 3. In general, bracket immersed in APF for 8 hours had the highest roughness values. The results of analysis of variance (ANOVA) showed a significant difference in $\mathrm{Ra}$ measurements between the studied groups $(\mathrm{P}<.05)$. Tukey post hoc tests revealed that utilization of APF for 8 hours provided a significantly higher $\mathrm{Ra}$ values than the other four studied protocols $(\mathrm{P}<.05)$. The later showed no significant differences between them $(\mathrm{P}>.05)$.

Regarding FL, storage in distilled water had the highest value, whereas storage in APF provided the lowest value. The other groups had intermediate values. Analysis of variance (ANOVA) indicated that there was a statistically significant difference in FL among the studied groups 
$(\mathrm{P}<.05)$. The results of Tukey test indicated no significant difference in FL among different studied groups $(\mathrm{P}>.05)$ except between brackets stored in distilled water and those stored in APF for 8 hours $(\mathrm{P}<.05)$.

\section{DISCUSSION}

The scope of the present study was to assess the effects of two prophylactic fluoride agents on polycrystalline ceramic self ligating bracket assembly. Brackets were immersed in APF or NaF solutions for 4 or 8 hours. These times were equivalent to approximately 8 or 16 months of one minute daily fluoride applications. ${ }^{11,26}$

In the present study, surface roughness was assessed using AFM. This type of microscope provides both quantitative and qualitative (3D) information about the surface morphology. In addition, it requires simple sample preparation. ${ }^{27}$ Results of the present study showed that, utilization of either fluoride solutions for 4 hours had no significant effect on Ra. This finding was in agreement with those of previous studies. ${ }^{26,28}$ Application of either fluoride solution for prolonged time ( 8 hours) exhibited different behaviors. NaF had no significant effect on Ra while APF significantly increased its value in comparison to the control group. In general with the use of fluoride, a chemical reaction between $\mathrm{F}^{-}$ions of fluoride with $\mathrm{Al}^{+3}$ ions of ceramic bracket leading to a strain of the alumina Al-O bonds. ${ }^{26}$ In addition, preferential dissolution of grain boundaries, ${ }^{29,30}$ where the interfaces between the particles are the weakest link. $^{23}$ This fluoride-related alumina damage could be more pronounced and exaggerated with the utilization of APF, acidic fluoride agent, for 8 hours. ${ }^{31-34}$

In the present study the fracture site was located either in the sliding ligature cap or at the ears of the brackets. The results of FL were in harmony with those of Ra. Application of either fluoride solutions for 4 hours had no significant effect on fracture resistance. This finding was in agreement with those of previous study evaluating the effect of prophylactic fluoride agents on fracture resistance of ceramic bracket tie wings. ${ }^{26}$ However, the FL values of the self ligating assembly were lesser than those reported for the tie wings of the traditional ceramic brackets. ${ }^{26}$ 
Egyptian

Orthodontic Journal

These differences could be attributed to differences in thickness and design of tie wing of traditional ceramic brackets and those of the self ligating assembly. In addition, differences in testing methodology could be another factor.

Increasing the time of APF application to 8 hours significantly reduced FL value. On the other hand, utilization of $\mathrm{NaF}$ for the same duration did not significantly alter FL values in comparison to the control group. This could be explained by immersion of brackets in acidic environment (APF) for long period (8hours) could lead to dramatic surface flaws and significant increase in alumina crack growth. ${ }^{31,34}$ Crack or imperfections allow for local concentration of the stresses and ceramic bracket fracture. ${ }^{22-24}$ However, as with any in vitro investigation, the protocol can not exactly simulate clinical situations. The brackets used in this study were new, and thus had no potential damage. However, there are numerous different factors, which probably lead to more stresses on the bracket in clinical situation such as mastication, temperature changes, closing/opening of the clip. Therefore, future studies are needed to investigate the effect of these factors on the mechanical properties of ceramic brackets.

\section{CONCLUSIONS}

Based on the results of the present study the flowing could be concluded:

- $\mathrm{NaF}$ could be used safely without adverse effects on polycrystalline ceramic self ligating brackets.

- Utilization of APF for long period significantly increased Ra and FL of polycrystalline ceramic self ligating bracket assembly.

\section{REFERENCES}

1. Gorelick L, Geiger AM, Gwinnett AJ. Incidence of white spot formation after bonding and banding. Am J Orthod. 1982;81:93-98.

2. Artun J, Brobakken BO. Prevalence of carious white spots after orthodontic treatment with multibonded appliances. Eur J Orthod. 1986;8:229-134. 
3. O'Reilly MM, Featherstone JDB. Demineralization and remineralization around orthodontic appliances-an in-vivo study. Am J Orthod Dentofacial Orthp.1987; 92:33-40.

4. Geiger AM, Gorelick L, Gwinnett AJ, Grisworld PG. The effect of fluoride program on white spot formation during orthodontic treatment. Am J Orthod Dentofacial Orthop. 1988;93:29-37.

5. Ogaard B. The prevalence of white spot lesion in 19-year-olds-a study on untreated and orthodontically treated persons 5 years after treatment. Am J Orthod Dentofacial Orthop. 1989;96:423-427.

6. Ogaard B, Rolla G, Arends J. Orthodontic appliances and enamel demineralization.1. Lesion development. Am J Orthod Dentofacial Orthop. 1988;94:68-73.

7. Ogaard B, Rolla G, Arends J, Tencate JM. Orthodontic appliances and enamel demineralization.2. Prevention and treatment of lesions Am J Orthod Dentofacial Orthop. 1988;94:123-128.

8. Probster L, Huttemann H. Effect of fluoride prophylactic agents on titanium surfaces. Int J Oral Maxillofacial Implants. 1992;7:390-394.

9. Reclaru L, Meyer JM. Effects of fluorides on titanium and other dental alloys in dentistry. Biomaterials. 1998;19:85-92.

10. Walker MP, White RJ, Kula KS. Effect of fluoride prophylactic agents on the mechanical properties of nickel-titanium-based orthodontic wires. Am J Orthod Dentofacial Orthp. 2005;127:662-669.

11. Walker MP, Ries DK, Kula K, Ellis M, Fricke B. Mechanical properties and surface characterization of beta titanium and stainless steel orthodontic wire following topical fluoride treatment. Angle Orthod. 2007;77:342-348.

12. Yaffe A, Zalkind M. The effect of topical application of fluoride on composite resin restoration. J Prosthet Dent.1981;45:59-62.

13. Wunderlich RC, Yaman P. In vitro effect of topical fluoride on dental porcelain. J Prosthet Dent. 1986;55:385-388.

14. Stolzenberg J. The Russell attachment and its improved advantages. Int J Orthod Dent Children. 1935; 21: 837-840. 
Egyptian

Orthodontic Journal

15. Russell JS. Current products and practice aesthetic orthodontic brackets. J Orthod. 2005;32:146-163.

16. Voudouris JC, Schisrenos C, Lackovic K, Kuftinec MM. Self-ligation esthetic brackets with low frictional resistance. Angle Orthod. 2010; 80:188-194.

17. Stefanos S, Secchi AG, Coby G, Tanna N, Mantef K. Friction between various self ligating brackets and arch wires couples during sliding mechanics. Am J Orthod Dentofacial Orthop. 2010; 138:463-467.

18. Kusy RP, Whitley JQ. Coefficients of friction for arch wires in stainless steel and polycrystalline alumina bracket slots. 1: the dry state. Am J Orthod Dentofacial Orthop. 1990;98:300-312.

19. Kusy RP, Whitley JO, Prewitt MJ. Comparison of frictional coefficients for selected arch wire-bracket slot combinations in dry and wet states. Angle Orthod. 1991;61:293-301.

20. Angolkar PV, Kapila S, Duncanson MG, Nanda RS. Friction between ceramic brackets and orthodontic wires of four alloys. Am J Orthod Dentofacial Orthop. 1990;98:499-506.

21. Doshi UH, Wasundhara A, Patil B. Static frictional force and surface roughness of various bracket and wire combination. Am J Orthod Dentofacial Orthop. 2011;139:74-79.

22. Scott GE. Fracture toughness and surface crakes - The key to understanding ceramic brackets. Angle Orthod. 1988;58:5-8.

23. Kusy RP. Morphology of polycrystalline aluminum brackets and its relationship to fracture toughness and strength. Angle Orthod. 1988;58:197-203.

24. Flores DA, Caruso JM, Scott GE, Jeiroudi MT. The fracture strength of ceramic brackets: a comparative study. Angle Orthod. 1990; 60:269-276.

25. Johnson G, Walker MP, Kula K. Fracture strength of ceramic bracket tie wings subjected to tension. Angle Orthod. 2005; 75:95-100.

26. Sanchez DJ, Walker MP, Kula K, Williams KB, Eick JD. Fluoride prophylactic agents effect on ceramic bracket tie-wing fracture strength. Angle Orthod. 2008;78:524-530. 
27. Choi S, Park K, Cheong Y, Kim H, Park Y, Park H. Changes in Ultrastructure and properties of bracket slots after Orthodontic treatment with bicuspid extraction. Scanning.2011:33:25-32.

28. Kula K, Josell S, Kula TJ. The effect of topical fluorides on ceramic brackets. Am J Orthod Dentofacial Orthop 1994; 106:513-517.

29. Fang Q, Sidky PS, Hocking MG. Effect of corrosion and erosion on ceramic materials. Corros Sci. 1997;39:511-527.

30. Barinov SM, Ivanove NV, Orlov SV, Shevchenko VJ. Influence of environment on delayed failure of alumina ceramics. Br Ceram Trans. 2000;99:39-42.

31. Kula K, Kula TJ. The effect of topical APF foam and other fluorides on veneer porcelain. Pediatr Dent . 1995;17:356-361.

32. Jones DA. Effect of topical fluoride preparations on glazed porcelain. J Prosthet Dent. 1985;53:483-484.

33. Dmirhanoglu ST, Sahin E. Effects of topical fluorides and citric acid on overglazed porcelain. Int J Prothodont. 1992;5:434-440.

34. Barinov SM, Fateeva LV, Ivanove NV, Orlov SV, Shevchenko VJ. Effect of acidic environment on subcritical crack growth in alumina ceramics. Scr Mater. 1998; 38:975-980. 